\title{
Long-term outcome of low contact stress total knee arthroplasty with different mobile bearing designs
}

\author{
GIUSEPPE SOLARINO, ANTONIO SPINARELLI, MASSIMILIANO CARROZZO, ANDREA \\ PIAZZOLLA, GIOVANNI VICENTI, BIAGIO MORETTI
}

Orthopaedic Section, Department of Basic Medical Sciences, Neurosciences and Sensory Organs, University of Bari "Aldo Moro", Bari, Italy

\begin{abstract}
Purpose: to evaluate the differences in clinical outcome and survivorship of three different mobile bearings for total knee arthroplasty.

Methods: a retrospective study was conducted in 60 patients ( 53 females, 7 males, mean age: 68 years and 5 months) each submitted to total knee replacement using one of the three different mobile bearings of the LCS system (Depuy Johnson \& Johnson, Warsaw, IN). The diagnosis was knee osteoarthritis in 57 cases and rheumatoid arthritis in three cases. Three different groups of 20 cases each were identified: total knee arthroplasties with mobile menisci (group 1); total knee arthroplasties with the rotating platform (group 2 ); and total knee arthroplasties with the anteroposterior glide platform (group 3). As regards the component fixation, 33 implants were cementless, three were cemented, and in 24 only the tibial component was cemented. The patella was not replaced.

Results: although the duration of follow-up differed between the three groups, the clinical and radiological results at final follow-up showed no revision of femoral and/or tibial components for mechanical or septic reasons, and no signs of impending failure. One meniscal bearing, showing polyethylene wear after 17 years, was successfully replaced.

Conclusions: the present retrospective study confirmed the long-term effectiveness of knee implants with mobile bearings, in which the congruity of the surfa-
\end{abstract}

\section{Corresponding Author:}

Giuseppe Solarino, MD

Orthopaedic Section, Department of Basic Medical

Sciences, Neurosciences and Sensory Organs, University of

Bari "Aldo Moro", Piazza Giulio Cesare 11, 70124 Bari, Italy

E-mail: giuseppe.solarino@ uniba.it ces makes it possible to overcome the problem of high contact stresses that may result in polyethylene wear and osteolysis; at the same time, these implants eliminate constraint forces thereby reducing the risk of mechanical loosening.

Level of evidence: Level III, retrospective comparative study.

Key words: knee arthroplasty, meniscal bearings, mobile bearing, rotating platform.

\section{Introduction}

Osteolysis induced by wear debris of ultra-high-molecular-weight polyethylene and the biological reaction to its particles in surrounding tissues has emerged as a significant problem after total knee arthroplasty (TKA). The generation of polyethylene wear and the subsequent development of osteolysis around a TKA are phenomena that result from a combination of patient-related, implant-related and surgical factors. Osteolysis can currently be considered the main reason for mid-term and long-term failure of a TKA. Implant - related factors are important to the outcome of a TKA - just as important as the patient's level of activity over time, which will affect the loads, and surgical factors, such as restoration of alignment and ligament balance. The optimal design of the articular bearing surface remains controversial, but nevertheless needs to be carefully considered, taking into account the stresses imparted on component-bone and modular tibial backside interfaces; the implant should be designed to grant mobility and stability, respecting the normal kinematics and avoiding peaks of stress on the polyethylene surface and constraint forces on the tibial component. In fact, although flat-on-flat designs allow 
extensive mobility, they increase the mechanical load, causing higher contact stress; on the other hand, implants with an intrinsic constraint transmit complex multidirectional stresses that act mainly on the tibial baseplate, resulting in its mechanical loosening in the intermediate term $(1,2)$. A TKA with a mobile bearing, maintaining the congruity of the surfaces and providing unconstrained mobility, may represent a good compromise able to significantly reduce polyethylene wear (3-6).

On the basis of these considerations, TKAs with mobile bearings have been used in our department since November 1993; initially the meniscal design (characterized by the presence of two curved tracks along the medial and lateral aspects of the metal tibial baseplate) was used; this was followed by the rotating platform, which only rotates about a central pivot without any movement in the frontal and sagittal planes, and finally the AP glide platform, which allows rotation and minimal controlled movement in the sagittal plane between the flat distal polyethylene surface and the highly polished proximal tibial tray. In a previous publication we have already reported the outcome of these implants at short term follow-up (7).

The purpose of the present study was to evaluate at long-term follow-up of the differences in clinical and radiological results of the total knee replacements performed using these three different mobile bearings. The hypothesis of the study was that total knee replacements performed using mobile bearings does not differ in clinical and radiological outcome according to the mobile bearing design at long-term follow-up.

\section{Methods}

We conducted a retrospective study of 60 patients (53 females, 7 males; mean age: 68.5 years, range $48-82$ years) who each underwent a total knee replacement using the LCS system (Low Contact Stress, Depuy, Johnson \& Johnson, Warsaw, IN) with one of the three different mobile bearing designs. The preoperative diagnosis was osteoarthritis in 57 cases and rheumatoid arthritis in 3. None of the patients was operated on bilaterally, and we therefore considered 60 implants: 33 cementless, 3 cemented, 24 hybrid (cementation only of the tibial component).
Three different groups were identified. Group 1 included 20 consecutive TKAs with mobile menisci. These were implanted in 16 females and 4 males (mean age 64 years, range: $48-82$ ) affected by knee osteoarthritis (18 cases) or rheumatoid arthritis ( 2 cases) who underwent the surgery between November 1993 and July 1994. In all cases both femoral and tibial components were fixed without cement. The posterior cruciate ligament was preserved in all the procedures.

Group 2 included 20 consecutive TKAs with the rotating platform. These were implanted in 18 females and 2 males (mean age 73 years, range: 62-81) with osteoarthritis (19 cases) or rheumatoid arthritis (1 case). They were operated on between January and May 1995. In 9 cases both components were cementless, in 10 only the tibial component was cemented, and in 1 case cemented femoral and tibial components were used. The posterior cruciate ligament, if present, was always sacrificed.

Group 3 included 20 consecutive TKAs with the AP glide platform. These were implanted in 19 females and 1 male (mean age 69 years, range: 57-75), all affected by osteoarthritis and operated on in the period between November 2000 and March 2001. In 4 cases cementless fixation of both components was performed, in 14 only the tibial component was cemented, and in 2 cases cemented femoral and tibial components were used. The posterior cruciate ligament was retained in all the procedures.

All the 60 cases were primary implants and no patient had a history of previous knee surgery, such as high tibial osteotomy, distal femoral osteotomy, or partial or total knee replacement.

The patella was never replaced; a pneumatic tourniquet was inflated before making the skin incision and was used during all the steps of the procedure; it was released after wound closure and elastic dressing. The articular drain was always removed on the second post-operative day; assisted and active motion was allowed thereafter. Partial weight-bearing with two crutches was started on the third post-operative day. Pharmacological and mechanical antithromboembolic prophylaxis was administered: low molecular weight heparin at a dose of $0.4 \mathrm{ml}$ per day subcutaneously, starting from the evening before the index operation and continuing for an average of 30 days, and an artero-venous foot pump in the recovery room, replaced 
with elastic stockings after removal of the drain. All the patients followed the same rehabilitation protocol. Radiological evaluation and clinical assessment with the Knee Society Score (KSS) (6) and Hospital for Special Surgery (HSS) score (8), respectively, were performed at baseline and at the most recent follow-up in December 2013. A statistical analysis with SPSS 11.0 software (SPSS Inc., Chicago, IL) was performed. Student's t test was used to perform pairwise comparisons for mean range of motion, mean tibial and femoral component survivorship, KSS and HSS score between the three groups. Significance was set for $p$ values $<0.05$.

\section{Results}

The overall follow-up duration differed between the three groups: up to 20 years in group 1, up to 18 years and 6 months in group 2, up to 13 years in group 3 . None of the implants needed revision for mechanical failure or sepsis; radiological evaluation showed stable fixation of all components, both femoral and tibial, without any sign of impending failure.

One meniscal bearing was successfully replaced 17 years after first surgery due to polyethylene wear of the lateral inlay on the femoral side (Fig. 1).

All the groups showed an improvement in the mean HSS score from the preoperative evaluation to the final follow-up: in group 1 it increased from 55 to 86 (average range of motion: $100^{\circ}$ ); in group 2 from 53 to 85 (average range of motion: $97^{\circ}$ ); in group 3 from 54.5 to 88 (average range of motion: $106^{\circ}$ ). Despite the evident discrepancy between the length of the final follow-up in the different groups, the results did not show statistically significant differences between them for average range of motion, survivorship, KSS and HSS score.

\section{Discussion}

The results reported in the present study were very similar to those obtained by Bistolfi et al. (9), who evaluated a consecutive series of 332 mobile-bearing, posterior-stabilized knee replacements in 249 patients at a mean follow-up of 76.3 months. The HSS score improved from 55 pre-operatively to 86 at the end of follow-up, a progressive radiolucent line appeared only in $1.2 \%$ of the knees, and the cumulative survival rate was $98.4 \%$ at 10 years, regardless of age, sex, disease severity, and patellar treatment (resurfaced in about $50 \%$ of the implants).

In the present study, cementless fixation of the components did not seem to affect the outcome. Similar findings were reported by Choy et al. (10) who, at a mean follow-up of 9.5 years, reported a $100 \%$ of survival rate of the femoral and tibial components in 186 consecutive primary LCS mobile-bearing TKAs performed by a single surgeon to treat knee $\mathrm{OA}$ in cases randomly assigned to undergo either cemented or cementless fixation of the tibial component.

Mobile-bearing designs were introduced in the United States by DePuy in the 1980s. The first ones were based on the meniscal-bearing concept, but they were soon followed by the rotating platform design. The purported advantage of the dual-surface articulation is that it provides a means of maintaining low rotational constraint and increasing the load bearing area (important for the durability of component fixation) while reducing tibiofemoral contact stresses and therefore addressing the problem of polyethylene wear. In fact, in mobile-bearing implants, the combination of increased congruity and unconstrained mobility has the effect of reducing contact stresses on the polyethylene surface to tolerable levels, avoiding peak stresses on the inlay (solving the problem presented by fixed flat-on-flat designs) and eliminating constraint forces on the tibial baseplate (solving the problem of highlyconformed prostheses with fixed inserts) (11). Otto et al. (12), in an experimental study conducted using the same implant with rotating platform that we used in our series, demonstrated that the polyethylene inlay follows the axial movements of the femoral condyles and can rotate about $6^{\circ}$; this results in maintenance of a high contact area, which is a desirable clinical behavior. Lacour et al. (13) recently demonstrated, in an in vivo three-dimensional kinematics study performed on a posterior-stabilized rotating-platform prosthesis, that tibial bearing rotation was maintained at 10 years postoperatively, although femoral component-polyethylene bearing rotation was reduced, suggesting that the overall kinematic performance of a mobilebearing implant is not negatively affected by time. 

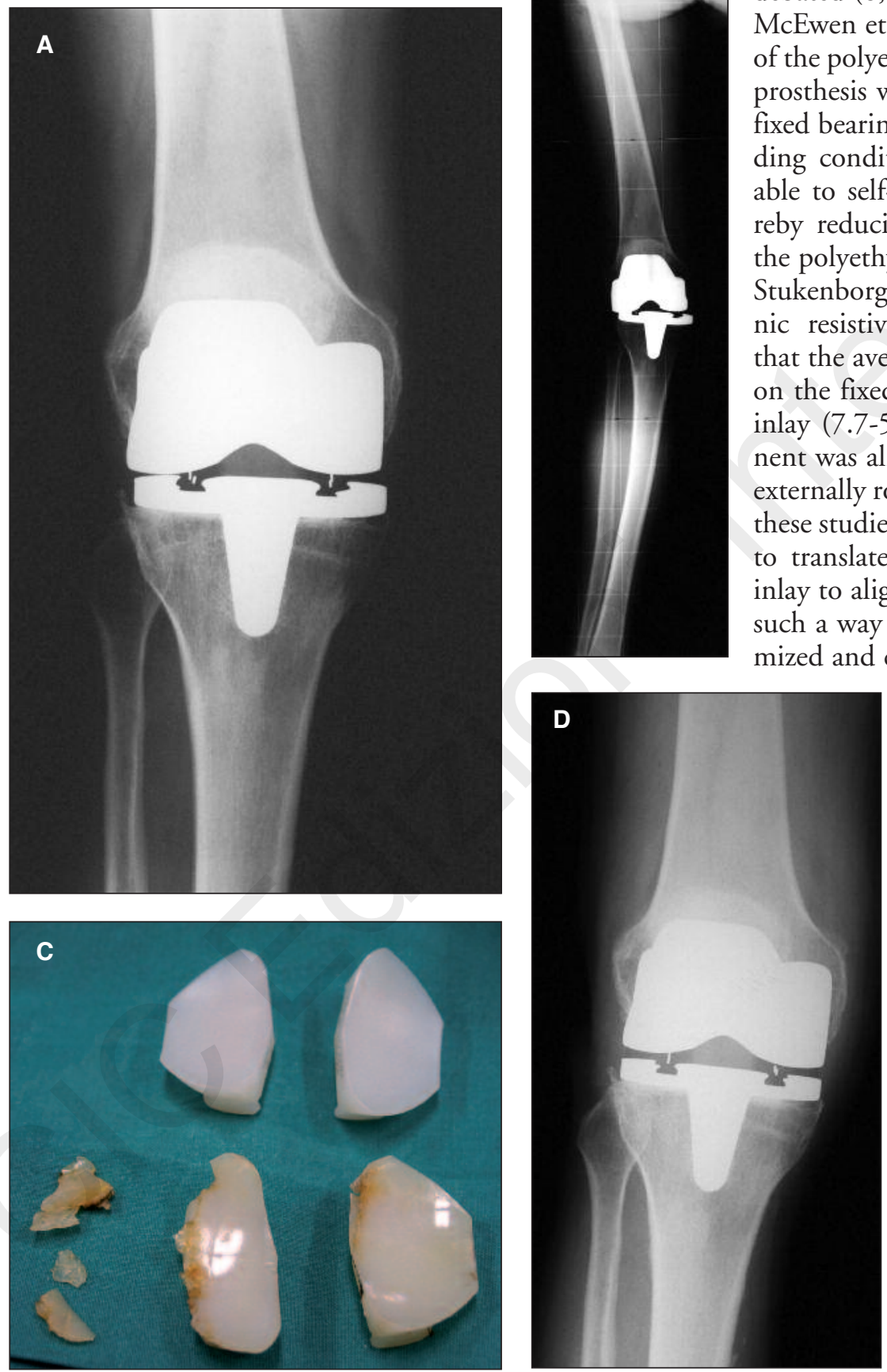

Fig. 1. A: Anteroposterior X-ray of a right total knee replacement with meniscal bearing after 17 years of survivorship shows loss of height in the lateral compartment due to wear of the latera bearing. B: Weight-bearing long-axis view shows the consequent valgus deformity. C: Intraoperative findings demonstrate fragments of the torn lateral meniscal bearing (above, the new devices to be used). D: Postoperative X-ray after replacement of the inlays.
Nevertheless the capacity of the femoral condyles and the inlay to reproduce the physiological posterior roll-back in the sagittal plane is still debated $(6,14)$.

McEwen et al. (15) showed that volumetric wear of the polyethylene is significantly lower in a knee prosthesis with a rotating platform rather than a fixed bearing device because, under dynamic loading conditions, the mobile bearing should be able to self-align to unidirectional motion, thereby reducing the forces acting tangentially on the polyethylene molecules.

Stukenborg-Colsman et al. (1), using an electronic resistive pressure-measuring sensor, found that the average peak contact stresses were higher on the fixed inlay $(21 \mathrm{MPa})$ than on the mobile inlay (7.7-5.3 MPa), whether the tibial component was aligned normally, or in an internally or externally rotated position. Ultimately, what both these studies suggest is that the ability of the inlay to translate on the tibial baseplate permits the inlay to align itself on the femoral component in such a way that the contact surface area is maximized and contact stresses are reduced.

Experimental data are confirmed by very satisfactory clinical results. Callaghan et al. (4) reported survivorship rates of between $94.6 \%$ and $100 \%$ in two series of patients with Oxford unicompartmental and LCS total knee replacements followed up for at least eight years.

Buechel and Pappas (3), comparing the six-year outcomes of cementless LCS total knee replacements, reported survivorship rates of $97.9 \%$ for the meniscal bearing and $98.1 \%$ for the rotating platform design. Ten years later the same authors, analyzing the results of cementless implants with a follow-up ranging from a minimum of 10 years to a maximum of 22 years, reported long-term survival rates of $83 \%$ at 16 years for the meniscal bearing and $88.3 \%$ at 18 years for the rotating platform design (16).

Such outstanding clinical results 
should be taken as confirmation of the validity of mobile-bearing implants and should overcome all the doubts about the possibility of increased wear due to the two sliding surfaces (femoral condyles-inlay and inlay-tibial baseplate). Moreover, micromotion of up to 25 micron with a cyclic axial load (17) and at least 100 micron with anteroposterior and mediolateral loads (18) has been recorded on the undersurface of the polyethylene between the tibial insert and baseplate in several modular fixed-bearing implants; this could create an undesirable second interface subject to backside wear $(2,19)$.

Nevertheless, the theoretical superiority of mobile bearings has not been reflected in better clinical performances, in terms of outcomes and survivorship, compared with their fixed counterparts.

Jacobs et al. (20), aiming to assess the differences in active flexion between patients who had undergone a total knee arthroplasty with a mobile or a fixed, cruciate retaining, bearing, enrolled 92 patients from one center using block-stratified, random allocation. Outcome parameters were flexion, both active and passive, and Knee Society score: no short-term differences in active flexion between fixed bearing and mobile bearing were found and the clinical Knee Society score was comparable between the two bearing groups.

In their evidence-based data review, Post et al. (21) found articles that tracked long-term (a minimum 10 years of follow-up) survivorship of fixed-bearing or mobile-bearing total knees, addressed functional outcome, or involved direct comparison of mobile-bearing and fixed-bearing prostheses. In summary, the authors demonstrated that at a follow-up of 12 to 23 years the available evidence does not point to the superiority of one design over another in terms of function and that there is no difference in survivorship between mobile-bearing and fixed-bearing designs, both being capable of producing excellent long-term results and clinical outcomes if properly implanted.

Wen et al. (22) conducted a systematic review and meta-analysis to evaluate whether mobile-bearing knee prostheses offer clinical and radiographic advantages over fixed-bearing knee prostheses. In the 15 studies involving 1,950 knees that they were able to identify (searching PubMed, EMBASE, Ovid and the Cochrane Central Register of Controlled Trials from January 1979 to June 2010) they did not find any statistically significant difference in terms of clinical sco- res, patient preference, radiolucent lines around the implant and prosthesis-related complications. They concluded that the mobile-bearing implant design, compared with the fixed-bearing implant design, improves outcomes only theoretically, given that the anticipated effectiveness has not yet been verified in current clinical practice.

These data were confirmed in a prospective randomized study conducted by Bailey et al. (23) in which no difference in clinical outcome, in 331 cases at two-year follow-up, was found between patients with rotatingplatform versus fixed-bearing posterior cruciate-retaining knee arthroplasties.

Finally, a randomized controlled trial in which a total of 116 surgeons in 34 centers examined the clinical effectiveness and cost-effectiveness of some aspects of knee replacement surgery, including the type of bearings (mobile or fixed to the tibial component), showed no definite advantage or disadvantage of mobile bearings in terms of clinical scores, quality of life, reoperation and revision rates or cost-effectiveness (24). In conclusion, our retrospective study confirmed the long-term effectiveness of knee implants with mobile bearings, in which the congruity of the surfaces makes it possible to overcome the problem of high contact stresses that may result in polyethylene wear and osteolysis; at the same time, these implants eliminate constraint forces thereby reducing the risk of mechanical loosening.

\section{References}

1. Stukenborg-Colsman C, Ostermeier S, Hurschler C, et al. Tibiofemoral contact stress after total knee arthroplasty: comparison of fixed and mobile-bearing inlay designs. Acta Orthop Scand. 2002;73:638-646.

2. Wasielewski RC, Parks N, Williams I, et al. Tibial insert undersurface as a contributing source of polyethylene wear debris. Clin Orthop Relat Res. 1997;345:53-59.

3. Buechel FF, Pappas MJ. Long-term survivorship analysis of cruciate-sparing versus cruciate-sacrificing knee prostheses using meniscal bearings. Clin Orthop Relat Res. 1990;260: 162-169.

4. Callaghan JJ, Squire MW, Goetz DD, et al. Cemented rotating-platform total knee replacement. A nine to twelve-year follow-up study. J Bone Joint Surg Am. 2000;82:705-711.

5. Cartier P, Landreau P, Sanouiller JP. Résultats des prothèses totales de genou LCS à plateau rotatoire. Rev Chir Orthop. 1997;83:32-33.

6. Ewald FC. The Knee Society total knee arthroplasty roentgenographic evaluation and scoring system. Clin Orthop Relat Res. 1989;248:9-12.

7. Solarino G, Luca A, Marzo L, et al. Comparison among total 


\section{(ont}

knee arthroplasties with a mobile bearing: menisci versus rotating platform versus AP glide platform. Chir Organi Mov. 2008;92: 79-83.

8. Insall JN, Dorr LD, Scott RD, et al. Rationale of the Knee Society clinical rating system. Clin Orthop Relat Res. 1989;248:13-14.

9. Bistolfi A, Lee GC, Deledda D, et al. NexGen(®) LPS mobile bearing total knee arthroplasty: 10-year results. Knee Surg Sports Traumatol Arthrosc. 2014 [Epub ahead of print].

10. Choy WS, Yang DS, Lee KW, et al. Cemented versus cementless fixation of a tibial component in LCS mobile-bearing total knee arthroplasty performed by a single surgeon. J Arthroplasty. 2014;doi: 10.1016/j.arth.2014.03.006.

11. Buechel FF Sr, Buechel FF Jr, Pappas MJ, et al. Twenty-year evaluation of meniscal bearing and rotating platform knee replacements. Clin Orthop Relat Res. 2001;388:41-50.

12. Otto JK, Callaghan JJ, Brown TD. Mobility and contact mechanics of a rotating platform total knee replacement. Clin Orthop Relat Res. 2001;392:24-37.

13. Lacour MT, Sharma A, Carr CB, et al. Confirmation of longterm in vivo bearing mobility in eight rotating-platform TKAs. Clin Orthop Relat Res. 2014 [Epub ahead of print].

14. Insall JN Adventures in mobile-bearing knee design: a midlife crisis. Orthopedics. 1998;21:1021-1023.

15. McEwen HM, McNulty DE, Auger DD, et al. Wear studies of the LCS. In: Hamelynck K, Stiehl J (eds) LCS® Mobile Bearing Knee Arthroplasty. Berlin Heidelberg. Springer. 2002;67-80.

16. Buechel FF The LCS Story. In: Hamelynck K, Stiehl J (eds)
LCS® Mobile Bearing Knee Arthroplasty, Berlin Heidelberg. Springer. 2002;19-25.

17. Wasielewski RC. The causes of insert backside wear in total knee arthroplasty. Clin Orthop Relat Res. 2002;404:232246.

18. Parks NL, Engh GA, Topoleski LD, et al. Modular tibial insert micromotion: a concern with contemporary knee implants. Clin Orthop Relat Res. 1998;356:10-15.

19. Ash HE, Scholes SC, Parkin R, et al. Relative movements between Kinemax Plus tibial inserts and the tibial base-plates. Proc Inst Mech Eng H. 2003;217:99-104.

20. Jacobs WC, Christen B, Wymenga AB, et al. Functional performance of mobile versus fixed bearing total knee prostheses: a randomised controlled trial. Knee Surg Sports Traumatol Arthrosc. 2012;20:1450-1455.

21. Post ZD, Matar WY, van de Leur T, et al. Mobile-bearing total knee arthroplasty: better than a fixed-bearing? J Arthroplasty. 2010;25:998-1003.

22. Wen Y, Liu D, Huang Y, Li B. A meta-analysis of the fixedbearing and mobile-bearing prostheses in total knee arthroplasty. Arch Orthop Trauma Surg. 2011;131:1341-1350.

23. Bailey O, Ferguson K, Crawfurd E, et al. No clinical difference between fixed- and mobile-bearing cruciate-retaining total knee arthroplasty: a prospective randomized study. Knee Surg Sports Traumatol Arthrosc. 2014 [Epub ahead of print].

24. Murray DW, MacLennan GS, Breeman S, et al. A randomised controlled trial of the clinical effectiveness and cost-effectiveness of different knee prostheses: the Knee Arthroplasty Trial (KAT). Health Technol Assess. 2014;18:1-235. 